\title{
A Hybrid Radio Frequency and Broadcast Visible Light Communication System*
}

\author{
Michael B. Rahaim ${ }^{\dagger}$, Anna Maria Vegni ${ }^{\S}$, and Thomas D.C. Little ${ }^{\dagger}$ \\ ${ }^{\dagger}$ Department of Electrical and Computer Engineering, \\ Boston University, Boston MA, USA \\ $\{$ mrahaim,tdcl $\} @ b u . e d u$ \\ $\S$ Department of Applied Electronics, \\ University of Roma Tre, Rome, Italy \\ amvegni@uniroma3.it
}

September 6, 2011

MCL Technical Report No. 09-06-2011

\begin{abstract}
Wireless network data consumption is experiencing drastic increases due to growing demands of mobile services and applications. Deployed networks using Radio Frequency (RF) communications are characterized by a shared medium, limited available spectrum and limited ability to scale with increasing demand. Directional communications, including Visible Light Communications (VLC), can limit contention in controlled environments and provide scalability through spatial reuse of the medium. This solution can provide massive aggregate data capacity in indoor scenarios if properly distributed.

In this paper we propose an indoor hybrid system that integrates WiFi and VLC luminaries. Our system utilizes $(i)$ broadcast VLC channels to supplement RF communications and (ii) a handover mechanism between WiFi and VLC to dynamically distribute resources and optimize system throughput. This approach offers the bandwidth density benefits of VLC, provides a non-intrusive RF back-channel, maintains compatibility with conventional RF devices, and demonstrates excellent scalability. Analytical and simulation results show improvements in aggregate performance (i.e., throughput and delay) of the hybrid, when compared to either system acting alone.
\end{abstract}

*In Proc. of 2nd IEEE Globecom 2011 Workshop on Optical Wireless Communications, December 5 - 9, 2011, Houston, Texas, USA. This work is supported primarily by the Engineering Research Centers Program of the National Science Foundation under NSF Cooperative Agreement No. EEC-0812056. Any opinions, findings, and conclusions or recommendations expressed in this material are those of the author(s) and do not necessarily reflect the views of the National Science Foundation. 


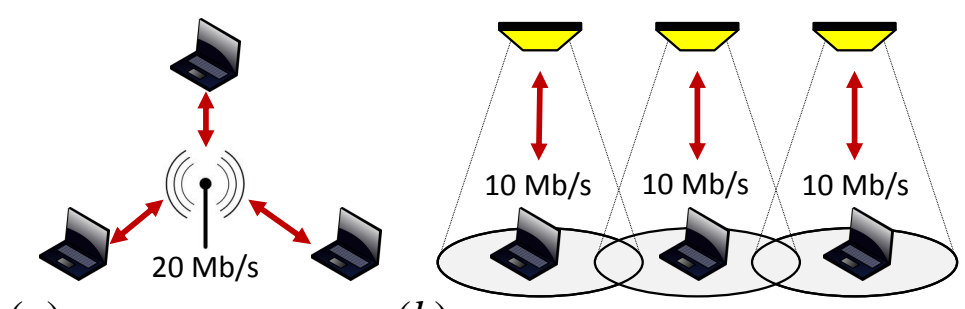

(a)

(b)

Figure 1: Bandwidth density of (a) RF and (b) VLC systems.

\section{Introduction}

Annual global IP traffic is expected to approach the zettabyte threshold by the end of 2015, four times the annual traffic from 2010. Forecasts expect traffic from wireless devices to exceed that from wired devices, and indicate that Internet video will account for $62 \%$ of the overall traffic [1]. As IP data traffic trends towards increased wireless usage and video streaming, the limitations of deployed radio frequency technologies will become evident.

Visible Light Communication (VLC) is emerging as a viable means to overcome the crowded radio spectrum for highly-localized communication systems [2]. VLC supports data communication and illumination in indoor environments where new energy-efficient LED materials and devices will replace old incandescent and fluorescent lighting. Among multiple advantages, VLC uses a vast unregulated and free spectrum [3]. Light signals also have the ability to be directed and sequestered allowing a VLC-enabled environment to transmit data in small cells and achieve high bandwidth densities $\left(\mathrm{Mb} / \mathrm{s} / \mathrm{m}^{2}\right)$. This includes cells in adjacent rooms or in a single room [4]. Figure 1 compares $a$ ) an RF channel in which three consumers share the $20 \mathrm{Mb} / \mathrm{s}$ bandwidth, to $b$ ) a VLC-enabled environment in which three consumers utilize individual $10 \mathrm{Mb} / \mathrm{s}$ VLC channels, providing $30 \mathrm{Mb} / \mathrm{s}$ of aggregate bandwidth.

Additionally, heterogeneous networks - Optical Wireless (OW) and Radio Frequency (RF) can co-exist allowing the benefits of VLC to enhance traditional RF systems by increasing system bandwidth. In this paper we propose an indoor hybrid system, comprised of traditional IEEE 802.11a/g technology and VLC links, in which directional broadcast VLC channels are exploited to supplement conventional RF channels. Such a system can provide additional bandwidth to account for increases in Internet traffic and offer the bandwidth density benefits to alleviate congestion in scenarios where multiple high data rate video streams are requested in close proximity. For example, WiFi congestion in hotels can be alleviated by providing non-interfering VLC channels in each room. Similarly, WiFi congestion issues in mass transportation (i.e., bus, subway, airplane) can be improved with the provision of separate VLC channels from each overhead light.

The paper is structured as follows. Section 2 describes current efforts in VLC. In Section 3 we investigate related works dealing with indoor integrated systems, which opportunistically exploit both RF and OW technology. Section 4 introduces the proposed asymmetric hybrid system as a standalone, and in cooperation with a bidirectional RF channel. Section 5 provides an analytical model and simulation results of the proposed system. Conclusions are drawn in Section 6. 


\section{Visible Light Communication}

Optical communications, including VLC, are typically implemented by Intensity Modulation and Direct Detection (IM/DD) [5]. VLC systems described in recent literature use approaches such as equalization, blue filtering and complex modulation to achieve data rates up to $500 \mathrm{Mb} / \mathrm{s}[6,7]$. However, approaching such rates with practical links remains a challenge; the demonstrated link is done at short range $(30 \mathrm{~cm})$ with off-line processing [7].

Although bidirectional VLC systems offer bandwidth density benefits and potential for higher data rates as an alternative to RF, some issues limit their marketability. These include intrusive uplink channels that produce an unpleasant irradiance from the user device, and user acceptance, because VLC faces challenges in displacing market incumbent RF systems which are considered sufficient and widespread.

The back-channel issue can potentially be addressed by low-powered VLC; however the illumination would still be visible to the user. Alternatively, the uplink channel can be addressed with an IR or RF link to each VLC transmitter. This asymmetric concept provides full connectivity at each VLC device meaning that each device must maintain a receiver and higher-level network connection.

To alleviate the issue of user acceptance, integrated systems allow consumers to maintain use of existing RF communications while benefitting from an additional OW channel. This concept has been explored as broadcast OW "hotspots" where RF communication provides individual channel control and uplink for each OW terminal [8].

\section{Related Work}

In this section we present recent related work on the use of VLC together with other pre-existing technologies, in order to enhance network performance and meet user Quality-of-Service (QoS) requirements.

Many authors have discussed how VLC and RF systems are complementary technologies [9]. VLC can deliver high capacity to multiple users within a small cell through the use of diffuse and spotlighting coverage while an RF system can produce a much larger cell at lower capacity. The heterogeneous nature of such technologies suggests scenarios where both systems would benefit from being used in combination.

Indoor environments, like offices, homes, and public common areas, are the best candidates to host heterogeneous networks (i.e., OW and RF). For example, VLC systems can be deployed to establish discrete high-capacity lighting cells, each covering a limited number of desks, while a single WiFi system can be deployed to cover the entire office. The use of both technologies relies on the growing user demand to be seamlessly connected to the Internet, while keeping high QoS levels and avoiding network congestions and delays.

Integrated optical and radio networks can be opportunistically chosen by both static and mobile users. The connectivity switching should be an automatic and seamless mechanism - called vertical handover (VHO) - from one wireless technology to the other, as users move throughout the room, thus maintaining a connected link at all times. Traditionally, handover is a process adopted by cellular systems (i.e., GSM, UMTS) to avoid service disconnections due to mobility. Particularly, a horizontal handover allows connectivity switching whenever a mobile user moves from one 


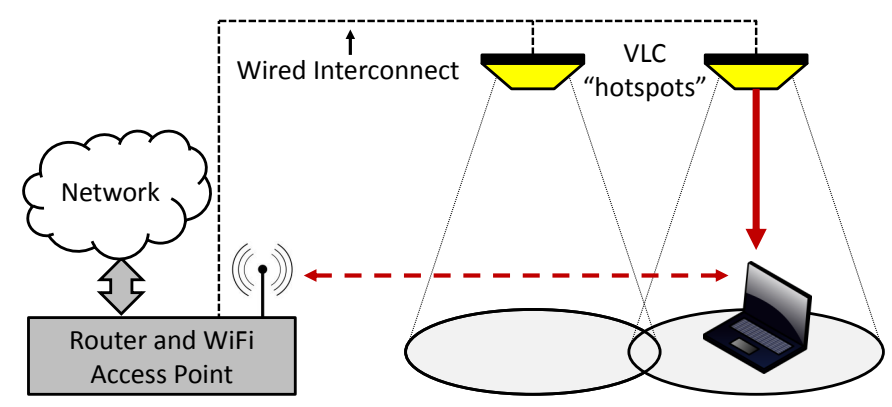

Figure 2: Proposed Broadcast WiFi-VLC Hybrid System.

access point to another without changing the service access network [10], while a vertical handover works in heterogeneous environments, where mobile users are moving among different networks.

In [11], a fuzzy logic-based vertical handover decision algorithm is proposed for an integrated WiFi-IR system. Combining the benefits of both technologies, optimal handovers occur for short and long-period interruptions. Rufo et al. [12] investigate the potential of a combination of VLC and WiFi system for an indoor broadcasting service. Their prototype consists of visible white LED lamps that achieve $2 \mathrm{Mbps}$ bit rates. Although this work is very promising, it still represents a feasibility study and leaves an open issue for future efforts.

In this paper we investigate the criteria for vertical handover in a hybrid system comprised of broadcast VLC hotspots and WiFi connectivity with a goal to avoid service disconnections and to optimally distribute available resources among users. We aim to demonstrate the benefit of moderate data rate VLC systems as a supplement to RF-based WiFi.

\section{Proposed system}

In this section we define our proposed hybrid system, in which the data link layer observes a Basic Service Set (BSS) containing multiple broadcast VLC hotspots within a wider range RF channel following the IEEE 802.11 standard. The static hybrid system and integration with a bidirectional RF channel are described in the following subsections.

\subsection{Broadcast VLC Hybrid}

The BSS for this system consists of multiple Mobile Terminals (MTs) equipped with VLC receivers and RF transceivers, as well as a single Access Point (AP) maintaining a RF transceiver and one or more broadcast VLC hotspots. Figure 2 shows the conceptual system utilizing two hotspots. The router maintains knowledge of the available hotspot for each MT and coordinates downlink traffic accordingly. If multiple MTs share the same downlink channel, the bandwidth is divided. Uplink occurs on the RF channel where medium access control is handled by the IEEE 802.11 Distributed Coordination Function (DCF) [13].

In order to maintain a listing of hotspots corresponding to each station, users must be able to identify the hotspots in range and forward that information to the AP. VLC hotspots may broadcast a node ID through either a time multiplexed frame or through a selective frequency channel. In this 
scenario horizontal handover consists of transfer between VLC hotspots in a single BSS or between $R F$ channel's as a MT moves from one BSS to another.

\subsection{RF and Hybrid Integration}

The benefit of utilizing an IEEE 802.11 compatible uplink channel is that it allows for a simple integration of the hybrid system and a bidirectional RF channel. In an integrated system, a MT is able to maintain full connectivity even if a VLC hotspot is unavailable, as long as the MT is in range of the RF channel. This allows the system to compensate for Line-Of-Sight (LOS) blockage and out-of-range VLC interruptions [14], by transferring downlink traffic from a VLC hotspot to the RF channel. This task is supported by a reactive vertical handover mechanism.

A reactive $\mathrm{VHO}$ requires a MT to initiate a handover procedure before breaking the connectivity with the VLC hotspot. Generally, in VLC systems the handover decision can be taken on the basis of a decrease in Received Signal Intensity (RSI) below a fixed threshold, mainly due to No-LOS conditions. However, if the connection between a MT and VLC hotspot breaks prior to handover, the MT can reestablish connection through the RF channel. Additionally, downlink traffic can be dynamically distributed between the RF and VLC channels in order to optimize the system throughput. Again, a reactive VHO mechanism is used to enhance network performance; each MT maintains its own QoS level and network congestions are limited. The high level description of the VHO algorithm is described below:

1. System Access: When joining the system, a MT should connect to the RF channel, assumed to be available;

2. VLC hotspot discovery: The MT observes the environment for an available VLC hotspot. If a hotspot is available, the user forwards the VLC node ID to the AP which maintains the collection of users in range of each VLC node. All channel control communication should be handled on the RF channel;

3. Handover assessment: Based on user requirements, the MT can immediately request transfer of downlink traffic to an available VLC hotspot (handover initiation) or can observe the QoS level of the RF channel and react accordingly;

4. Channel monitoring: Once connected to the hotspot, the MT should monitor RSI and request transfer of downlink traffic back to the RF channel if the RSI parameter drops below a fixed threshold. Additionally, the MT should monitor channel capacity and request transfer of downlink traffic back to the RF channel if the hotspot becomes overloaded.

\section{Performance analysis}

In this section we analyze the static system in order to offer an idea of the potential benefits offered by the proposed hybrid model. We provide analysis of the ideal downlink channel, as well as results of a simulated system. Throughout this section, we distinguish traffic as either interactive (low data rate) or streaming (high data rate). We assume $25 \mathrm{Mb} / \mathrm{s} \mathrm{WiFi}$ and $50 \mathrm{Mb} / \mathrm{s} \mathrm{VLC}$ capacity as well as $0.3 \mathrm{Mb} / \mathrm{s}$ and $9.8 \mathrm{Mb} / \mathrm{s}$ request rates for interactive and streaming users, respectively. 
Table 1:

\begin{tabular}{c|c|c}
\multicolumn{3}{c}{ Parameters for Downlink Channel Analysis } \\
\hline \hline Channel Rate & Packet Size & Arrival Rate \\
\hline$R^{(W)}=25 \mathrm{Mb} / \mathrm{s}$ & $M_{I}=8 \mathrm{~kb}$ & $\lambda_{I}=37.5$ \\
$R^{(V)}=50 \mathrm{Mb} / \mathrm{s}$ & $M_{S}=16 \mathrm{~kb}$ & $\lambda_{S}=612.5$ \\
\hline
\end{tabular}

\subsection{Downlink Channel Analysis}

Our preliminary analysis observes downlink traffic for an office scenario in which the environment contains four non-contending VLC enabled light fixtures and a single WiFi channel covering the entire space. We analyze downlink traffic in three cases, such as:

- Case 1: Utilizing only the WiFi channel. We assume there is no contention on the channel and the entire WiFi capacity is available for downlink traffic;

- Case 2: Utilizing an asymmetric system where all downlink traffic is communicated through VLC. We assume users are evenly distributed amongst the VLC channels;

- Case 3: Utilizing a hybrid system with a simplified handover mechanism in which users are offloaded to a VLC channel once the WiFi channel has reached capacity.

The number of interactive and streaming users in the system are defined as $N_{I}, N_{S}$, respectively. We observe each channel as an M/D/1 queue and each user as a Poisson arrival stream with rate $\lambda_{I}, \lambda_{S}$ [packets/second] for interactive and streaming users, respectively. Transmission rate is dependent on the channel type (i.e., $R^{(W)}, R^{(V)}$ [Mbit/s] for WiFi and VLC, respectively) and packet size is dependent on user type (i.e., $M_{I}, M_{S}$ [bit] for interactive and streaming users, respectively). Table 1 collects values used for analysis.

Case 1 is observed as a single WiFi queue, i.e., $Q_{1}^{(W)}$, where all arrivals are presented. Case 2 assumes four VLC downlink channels, corresponding to four VLC queues, i.e., $Q_{2}^{\left(V_{x}\right)}$ with $x=$ $\{1,2,3,4\}$, each with an approximately equal distribution of the streaming user and interactive user arrival streams. Finally, Case 3 is observed as a single WiFi queue, i.e., $Q_{3}^{(W)}$, and four additional VLC queues, i.e., $Q_{3}^{\left(V_{x}\right)}$ with $x=\{1,2,3,4\}$. In this case, all interactive user arrival streams are presented to the WiFi queue, while streaming user arrival streams are presented to the WiFi queue until it becomes unstable; at that point all remaining streaming user arrival streams are equally distributed amongst the VLC queues.

By summation of Poisson streams, we obtain the following arrival rates for each queue in the $i$-th case with $i=\{1,2,3\}$

$$
\lambda_{i}^{(q)}=\lambda_{I, i}^{(q)} \cdot N_{I, i}^{(q)}+\lambda_{S, i}^{(q)} \cdot N_{S, i}^{(q)}
$$

where $q=\left\{W, V_{1}, V_{2}, V_{3}, V_{4}\right\}$ is the label of each queue described above; $N_{I, i}^{(q)}$ and $N_{S, i}^{(q)}$ represent the number of interactive and streaming user arrival streams at the specified queue for the $i$-th case.

The expected service time, i.e., $E\left[X_{i}^{(q)}\right][\mathrm{s}]$, for a packet in each queue is similarly defined as:

$$
E\left[X_{i}^{(q)}\right]=\frac{\lambda_{I, i}^{(q)} \cdot N_{I, i}^{(q)} \cdot M_{I}+\lambda_{S, i}^{(q)} \cdot N_{S, i}^{(q)} \cdot M_{S}}{\lambda_{i}^{(q)} \cdot R^{(q)}} .
$$




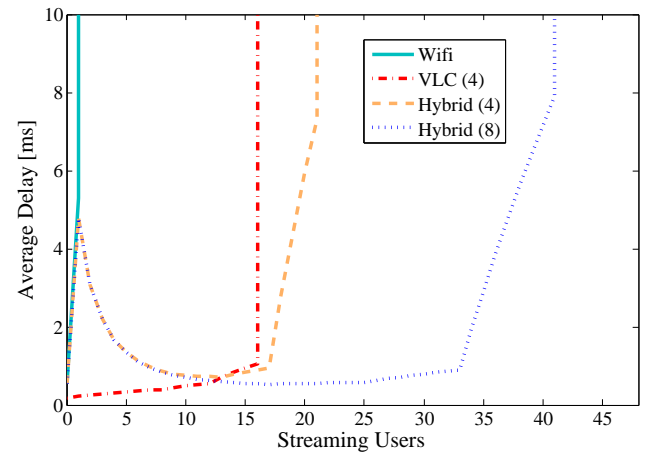

(a)

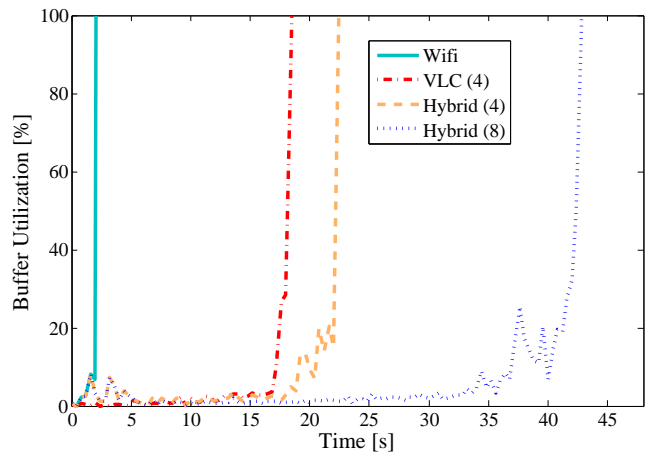

(b)

Figure 3: (a) Analytical results and (b) simulation results for downlink channel analysis of a system with 48 users.

Assuming a constant packet size (i.e., $M_{I}, M_{S}$ ), we can also determine the workload, i.e., $\rho_{i}^{(q)}$, the residual service time, i.e., $T_{R, i}^{(q)}[\mathrm{s}]$, and the expected queuing time, i.e., $E\left[T_{Q, i}^{(q)}\right][\mathrm{s}]$, for each queue, such as:

$$
\begin{gathered}
\rho_{i}^{(q)}=\lambda_{i}^{(q)} \cdot E\left[X_{i}^{(q)}\right], \\
T_{R, i}^{(q)}=\frac{\lambda_{i}^{(q)} \cdot E\left[X_{i}^{(q)}\right]^{2}}{2}, \\
E\left[T_{Q, i}^{(q)}\right]=\frac{T_{R, i}^{(q)}}{1-\rho_{i}^{(q)}} .
\end{gathered}
$$

It follows that the average delay of each queue, i.e., $D_{i}^{(q)}[\mathrm{s}]$, is the sum of the expected time spent queuing and the expected time spent in service, such as:

$$
D_{i}^{(q)}=E\left[T_{Q, i}^{(q)}\right]+E\left[X_{i}^{(q)}\right]
$$

The average delay of the WiFi system, i.e., $D_{W i F i}$, is the average delay of the WiFi queue from Case 1 . The average delay of the asymmetric, i.e., $D_{a s y m}$, and hybrid, i.e., $D_{h y b}$, systems are the weighted average of the delays from the queues in Case 2 and 3, respectively.

$$
\begin{gathered}
D_{W i F i}=D_{1}, \\
D_{\text {asym }}=\frac{\sum_{x=1}^{4}\left[\lambda_{2}^{\left(V_{x}\right)} \cdot D_{2}^{\left(V_{x}\right)}\right]}{\lambda_{I} \cdot N_{I}+\lambda_{S} \cdot N_{S}}, \\
D_{h y b}=\frac{\lambda_{3}^{(W)} \cdot D_{3}^{(W)}+\sum_{x=1}^{4}\left[\lambda_{3}^{\left(V_{x}\right)} \cdot D_{3}^{\left(V_{x}\right)}\right]}{\lambda_{I} \cdot N_{I}+\lambda_{S} \cdot N_{S}} .
\end{gathered}
$$

The analytical results of the downlink scenario are shown in Figure $3(a)$. We observe a constant total of 48 users with varied distribution between interactive and streaming users. Delay is depicted 
versus the number of streaming users for Cases 1, 2, and 3, labeled "WiFi", "VLC (4)", and "Hybrid (4)", respectively. Moreover, we show results of a fourth case, i.e., "Hybrid (8)", that is an extended version of Case 3 with eight VLC downlink channels. Analysis shows that Cases 1, 2 and 3 and the Hybrid (8) scenario become unstable at 2, 17, 22 and 42 users, respectively. When comparing the two hybrid scenarios, it can be seen that additional VLC channels provide additional aggregate system bandwidth, illuding to the scalability of the system.

In the hybrid situations, delay follows the curve of the WiFi only scenario until the total traffic becomes greater than the capacity of the WiFi channel. At this point, streaming users are offloaded to VLC channels and average delay improves until VLC channels begin to see delays greater than that of the WiFi channel. The 22nd and 42nd streaming user causes the first of the VLC links to become unstable in hybrid situations with four and eight VLC channels, respectively.

Simulation results are provided in Figure $3(b)$. We simulate the four cases described and observe the amount of buffered data at each transmitter as the number of streaming users increases (one user per second). Results are displayed as a percentage of the buffer space used by all active transmitters. As with the analytical results, we show that Cases 1,2 and 3 become unstable at 2, 17 and 22 streaming users, respectively. The Hybrid (8) scenario also validates the analytical results, showing instability at 42 users. In addition, increases in buffer utilization can be seen at times where analytical results predict a higher average delay.

\subsection{System Analysis}

We simulate the interaction between streaming traffic at an AP and congestive traffic from $n$ interactive users utilizing the WiFi channel. Streaming traffic is distributed between one or more VLC channels and a single WiFi channel in contention with the users. For each simulated case, we also discuss how a reactive vertical handover mechanism occurs, in order to $(i)$ optimally distribute resources in the hybrid system, and (ii) avoid quick and long-term disconnections. Regarding contention on the WiFi channel, we simulate the basic access mechanism of the IEEE 802.11 DCF as described in [15].

Figure $4(a)$ and $(b)$ show per channel and system downlink throughput results of a simulation with 10 contending users and 10 streaming users, for a single VLC channel and multiple VLC channels, respectively. We observe throughput as streaming users are offloaded from the WiFi channel to VLC.

In Figure $4(a)$, all users share a single VLC hotspot. The optimal distribution of users - where system throughput is maximized - occurs with $6-7$ users on the VLC channel and 3-4 users on the WiFi channel. At this point, both the VLC channel and WiFi channel are being fully utilized. In this case, the handover mechanism should occur whenever a VLC channel has the capacity to accept new users coming from the WiFi channel.

In Figure $4(b)$, each user observes a separate VLC hotspot. Provided that the requested data rate of each user is less than the bandwidth of the VLC link, the optimal distribution occurs when all users are utilizing their VLC link, leaving the WiFi medium solely for uplink traffic and showing the scalability and bandwidth density benefits of the system. As users are transferred to their VLC hotspot, they are utilizing an uncontended channel and providing additional system bandwidth. In this case, new users will connect to the WiFi network and immediately request transfer of downlink traffic to their VLC hotspot. A vertical handover to the WiFi network should be initiated only if a disconnection from a VLC channel occurs. 


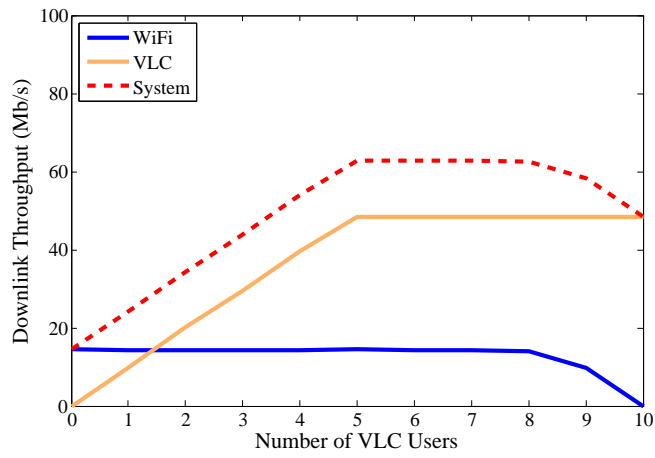

(a)

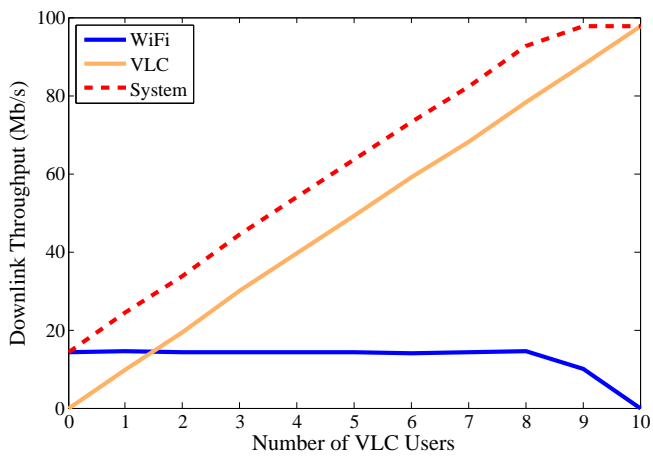

(b)

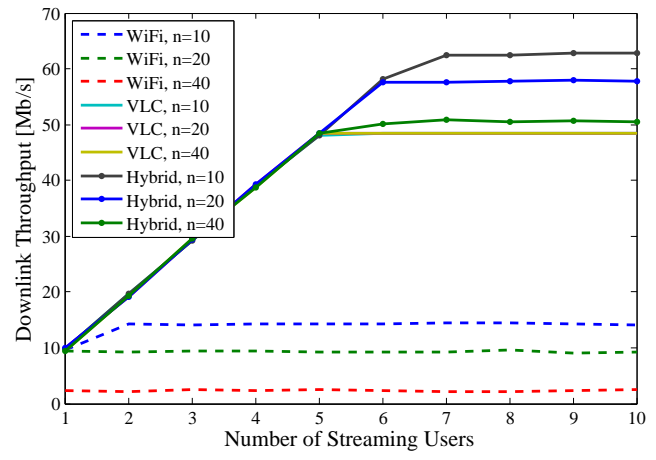

(c)

Figure 4: Effective downlink throughput for a hybrid system with ten contending users and ten streaming users with a single WiFi channel and $(a)$ one VLC channel, $(b)$ multiple VLC channels. (c) Throughput comparison for WiFi only, asymmetric VLC and hybrid systems with $n$ contending users.

In Figure $4(c)$, we compare an optimal hybrid system with a single VLC channel to a WiFi only and asymmetric VLC system. We show downlink throughput versus streaming traffic where 10, 20, and 40 users are contending for the WiFi channel. Observation shows that the hybrid has potential to perform better than either system acting alone. The asymmetric VLC system saturates near the VLC bandwidth independent of uplink traffic. In both the WiFi and hybrid systems the downlink traffic saturates dependent on the amount of uplink traffic; however the additional bandwidth of the VLC channel allows the hybrid to reach higher system throughput.

\section{Conclusions}

In this paper, we have proposed and analyzed a hybrid system that integrates directional broadcast VLC channels with an omnidirectional RF channel. Through analytical and simulated analysis, we have shown that our system provides additional aggregate capacity and alleviates contentions on the RF channel. The dynamic allocation of users between downlink channels has been investigated and we provided potential results of an optimal allocation scheme and potential VHO mechanism.

In summary, the proposed system represents an opportunity to bring VLC to market without acting as a competitor to conventional RF devices. Developing VLC systems that supplement 
WiFi allows the aggregate capacity to increase linearly as the number of VLC channels increases. This scalability offers benefits in overall system throughput that will be needed as we trend towards the forecasted increases in wireless network traffic.

\section{References}

[1] Cisco Visual Networking Index. "Forecast and Methodology, 2010-2015". San Jose, CA, June 1, 2011.

[2] J.B. Carruthers. Wireless Infrared Communications. Wiley Encyclopedia of Telecommunications, 2002.

[3] M. Kavehrad. "Sustainable Energy-Efficient Wireless Applications Using Light". IEEE Communications Magazine, 2010.

[4] T. Borogovac, M. Rahaim, and J. Carruthers. "Spotlighting for Visible Light Communications and Illumination". In Proc. of 1st IEEE Workshop on Optical Wireless Communications, IEEE GLOBECOM, 2010.

[5] J. Kahn and J. Barry. "Wireless Infrared Communications". Proc. of the IEEE, 85(2):265298, February 1997.

[6] H. Minh et al. "High-Speed Visible Light Communications Using Multiple-Resonant Equalization”. IEEE Photonics Technology Lett., 20(14), 2008.

[7] J. Vucic et al. "513 Mbit/s Visible Light Communications Link Based on DMT-Modulation of a White LED”. Journal of Lightwave Technology, 28(24):3512-3518, December 2010.

[8] D. O'Brien. “Cooperation in Optical Wireless Communications. Cognitive Wireless Networks, pages 623-634, 2007.

[9] D.J.T. Heatley, D.R. Wisely, I. Neild, and P. Cochrane. "Optical Wireless: The story so far". IEEE Communications Magazine, 36(12):72-74, 79-82, December 1998.

[10] G.P. Pollini. “Trends in Handover Design”. IEEE Communications Magazine, 34(3):82-90, 1996.

[11] J. Hou and D. O’Brien. "Vertical Handover Decision-Making Algorithm using Fuzzy Logic for the Integrated Radio-and-OW system". IEEE Trans. on Wireless Communications, 5(1):176-185, January 2006.

[12] J. Rufo et al. "Experimental Evaluation of Video Transmission through LED Illumination Devices". IEEE Trans. on Consumer Electronics, 56(3):1411-1416, August 2010.

[13] IEEE Standard for Wireless LAN Medium Access Control (MAC) and Physical layer (PHY) Specifications, Nov. 1997.

[14] J. Hou and D. O'Brien. "Polling Scheme for Indoor LOS Optical Wireless LAN". Electron. Lett., 39(10):794-795, 2003. 
[15] G. Bianchi. "Performance Analysis of the IEEE 802.11 Distributed Coordination Function". IEEE JSAC, 18(3), March 2000. 